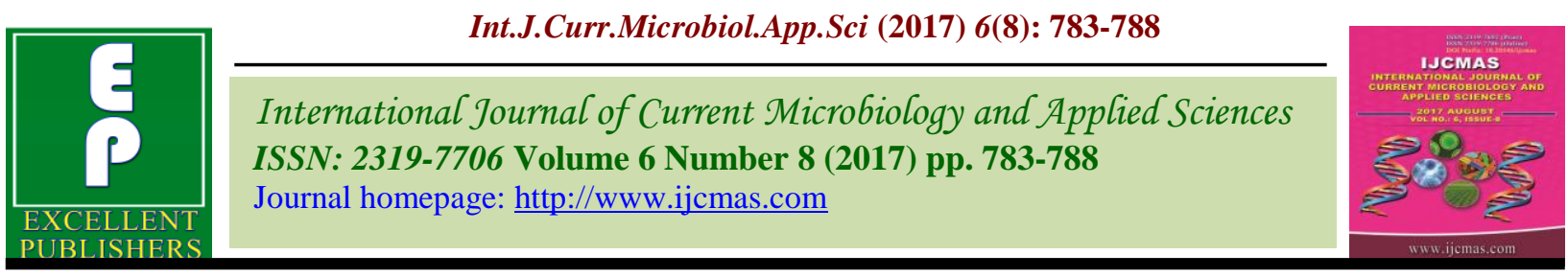

Original Research Article

https://doi.org/10.20546/ijcmas.2017.608.099

\title{
Performance of SD Bioline Syphilis 3.0 for the Diagnosis of Syphilis a UPFR in Immunology of CHU-JRA
}

\author{
Arinomenjanahary Rakotoarisoa ${ }^{1 *}$, Herinjaka Andriamandimbisoa ${ }^{1}$, \\ Toky Randriamahazo², Jaonoharimandimby Andrianavalona ${ }^{2}$, \\ Davidra Rajaonatahiana ${ }^{3}$ and Andry Rasamindrakotroka ${ }^{3}$ \\ ${ }^{1}$ Laboratory Bacteriology CHU-JRA Antananarivo, Madagascar \\ ${ }^{2}$ Laboratory of Immunology CHU-JRA Antananarivo, Madagascar \\ ${ }^{3}$ Department of Immunology Medecine Faculty Antananarivo, Madagascar \\ *Corresponding author
}

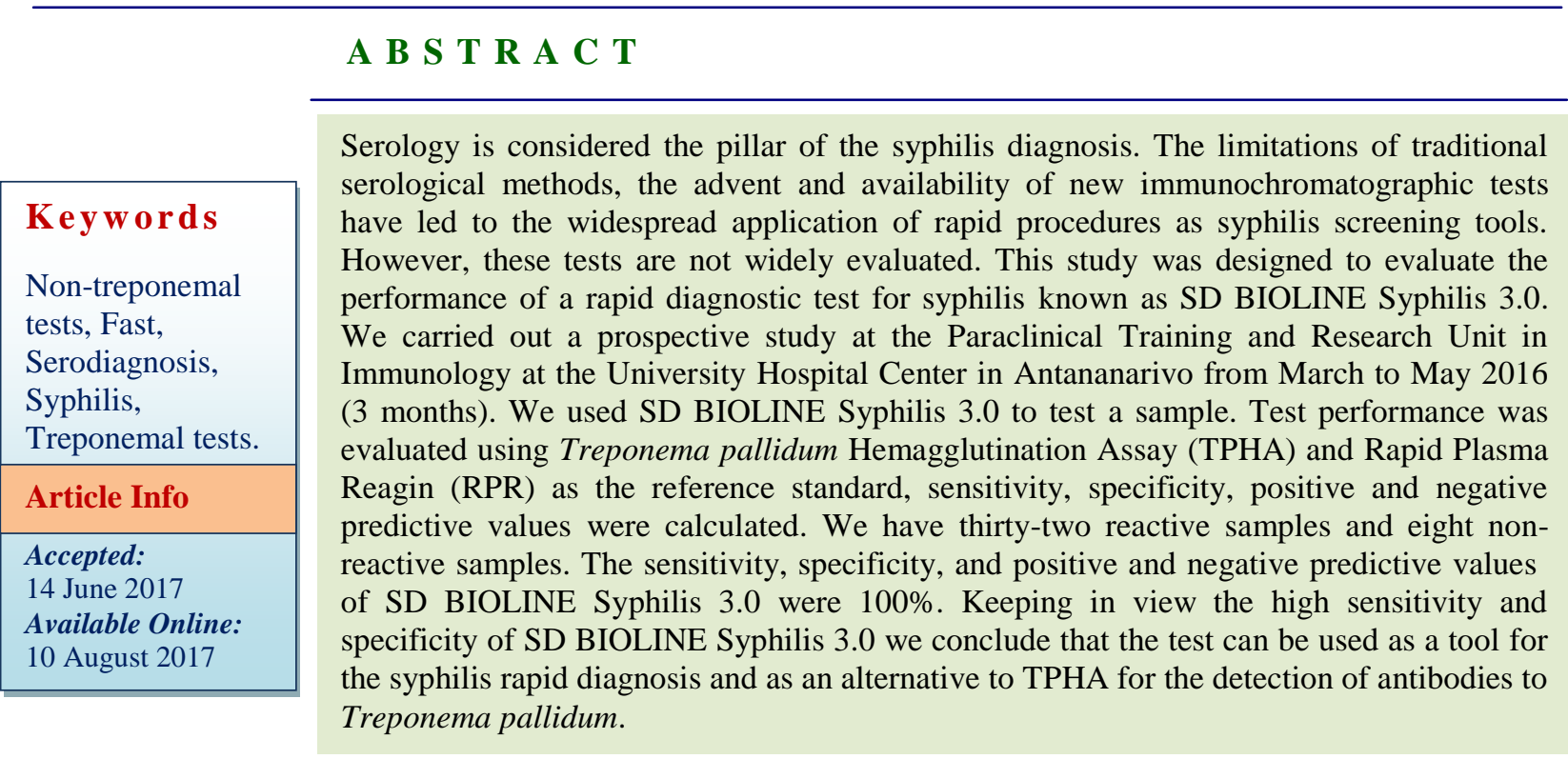

\section{Introduction}

Syphilis is a sexually transmitted infection (STI) caused by the bacterium Treponema pallidum ( $T$. pallidum), a disease that is becoming widespread. According to the WHO in 2012; 900000 women were infected by syphilis in the world. Furthermore, the inability of culture of the pathogenic microorganism in vitro and the limited availability of nucleic acid amplification techniques makes the diagnosis of this infection difficult [1].
Serology is therefore considered to be the pillar of the syphilis diagnosis. The serological diagnosis of syphilis is based with detection of the two types antibodies directed against the cardiolipin antigen and treponemal-specific antibodies [2, 3]. A major limitation of diagnosis is encountered with the use of tests based on antiphospholipid antibodies (non-treponemal test) giving false positive results $[4,5,6]$. It is therefore recommended to use non-treponemal tests 
such as VDRL and Rapid Plasma Reinforce (RPR) for screening tests and then confirmed by more specific treponemal tests such as $T$. pallidum hemagglutination Assay (TPHA) Fluorescent treponemal antibodies [7, 8, and 9]. False negative reactions due to the zone phenomenon are also seen with nontreponemal tests [10]. Moreover, the tests lack sensitivity in the latent stage of latent infection [11]. A major disadvantage of the laboratory procedures currently used for syphilis serodiagnosis, which require various materials (refrigeration, water bath, centrifuge, rotators, etc.); strict quality control measures and qualified individuals to perform the tests, as well as trained health professionals to read and interpret the results. In the stress parameters, laboratory infrastructure and facilities for the diagnosis of syphilis may not be widely available, and the delay in obtaining samples tested by reference laboratories may prevent timely initiation of treatment. This is reflected in the continued transmission of the disease to naïve or uninfected individuals. The current situation requires to need for rapid and reliable tests used for screening and confirmatory testing in all stages of syphilis. Rapid serological procedures offer a potential option with assured rapid availability of results generally $<15$ min and ease of use by healthcare professionals allowing on-site testing. The World Health Organization has established a diagnostic initiative for sexually transmitted diseases, the "ASSURED" criteria that define the characteristics of the rapid test ideal and a point of care test (Affordable, sensitive, specific, user- [12-14]. It is also available as an affordable, sensitive, specific, fast and robust device that is easy to use for free and available to those who need it. Several rapid $T$. pallidum recombinant antigens are now commercially available [15]. Despite the advantages that rapid tests offer over traditional laboratory methods for the diagnosis of syphilis, their diagnostic performance remains a concern and is still poorly documented. In this study, the authors evaluated the performance of SD BIOLINE Syphilis 3.0, a rapid immunochromatographic test that qualitatively detects antibodies against $T$. pallidum.

\section{Materials and Methods}

This prospective study was conducted at the Paraclinical Unit of Training and Research in Immunology at the University Hospital Center of Antananarivo, from March to May 2016 (3 months). This study was duly approved by the Head of Unit. Samples of consecutive blood samples received at the serology laboratory with syphilis screening were included in the analysis.

All reactive RPR sample were diluted serially to determine the antibody titer. Three different staff performed the analysis: the first technician who did not have access to the results of the screening test performed the TPHA according to the manufacturer's instructions in the kit. A second performed the TPHA and then the third laboratory technician performed the test on SD BIOLINE Syphilis 3.0 per the manufacturer's guidelines.

The results of the RPR, TPHA and SD BIOLINE Syphilis 3.0 assays were entered the Microsoft Excel sheet and discordant results by TPHA and SD BIOLINE Syphilis 3.0 were retested by both procedures before finally being recorded as positive or negative.

Calculation and estimation of the positive, negative predictive values of SD BIOLINE Syphilis 3.0 sensitivity and specificity were performed by comparing its performance with TPHA (reference technique). Sensitivity was calculated as true positive / (true positive + false negative) $\times 100$; Specificity as true negatives / (true negatives + false positives) $\mathrm{x}$ 
100; Negative predictive value as true negative / (true negative + false negative) $\times$ 100, and positive predictive value as true positive / (positive + false positives) x 100 .

\section{Results and Discussion}

We worked on 40 samples including 8 RPR negative and 32 RPR reactive samples, TPHA positive, and reactive with SD BIOLINE Syphilis 3.0. All non-reactive RPR sample (n $=8$ ) were negative both by TPHA and the rapid test. The performance of SD BIOLINE Syphilis 3.0 and RPR against TPHA is presented in table 1 .

We report the sensitivity and specificity of $100 \%$ values for SD BIOLINE Syphilis 3.0, relative to TPHA as the reference standard. A study in Tanzania found that the test was $79 \%$ sensitive and $96 \%$ specific [16]. In another study conducted in China, reported sensitivity and specificity values were $95.5 \%$ and $97.9 \%$, respectively [17].

Thus, bearing in mind the high sensitivity and specificity of SD BIOLINE syphilis 3.0, we conclude that the assay can be used both as a screening test and as an alternative to TPHA for the detection of antibodies directed against $T$. pallidum. The low sensitivity of immunochromatographic tests has been reported in previous studies, and the reason given was mainly the low levels of antibodies found in sera from patients with primary early syphilis [18].
Discordant results between TPHA and SD BIOLINE Syphilis 3.0 (TPHA BIOLINE Syphilis 3.0 negative and SD positive sera) may occur, observed in another study that could be explained by the fact that many treponemal antigens are exposed during the TPHA test. Therefore, some cross-reactivity with other organisms may occur. In addition, as some researchers have hypothesized, although the recombinant antigens used in rapid tests are specific to $T$. pallidum, the post-translational transformation of these proteins in vivo or the lack of a genetically determined response or limited reactivity to these antigens may lead a lack of reactivity, even in the presence of the disease [19].

SD BIOLINE Syphilis 3.0 is a quick one-step test that is easy to perform, read and interpret. In addition to serum samples, the test is also compatible with whole blood and plasma. Therefore, the test does not require any pretreatment of specimens or any laboratory or qualified technical personnel to perform the test procedure. This makes the test an ideal on-site option in screening in health facilities without laboratory facilities.

Furthermore, since the $T$. pallidum-specific antibody test does not give a false positive and the test can also be used as an alternative to TPHA to confirm reactivity in nontreponemal tests. The rapid availability of results with the use of this test would also ensure rapid treatment of reactive patients in their first visit to health care services.

Table.1 Performance of SD BIOLINE Syphilis 3.0 in comparison with TPHA * (standard reference) and RPR **

\begin{tabular}{|c|c|c|c|}
\hline Résults & RPR & TPHA & SB BIOLINE Syphilis3.0 \\
\hline Positif & 32 & 32 & 32 \\
\hline Négatif & 8 & 8 & 8 \\
\hline Sensibility & $100 \%$ & $100 \%$ & $100 \%$ \\
\hline Specificity & $100 \%$ & $100 \%$ & $100 \%$ \\
\hline
\end{tabular}

TPHA*: Treponema pallidum hemagglutination assay;

RPR**: Réagine plasmatique rapide 
The test, however, faces some shortcomings. An important constraint for its application as a screening tool is that the treponemalspecific antibodies detected by the test are conserved for years and thus cannot distinguish past treated infections from recent or active infections [20]. This implies that many previously infected and treated people would also be captured by SD BIOLINE syphilis 3.0, especially in high-prevalence settings, leading to unnecessary treatment of patients.

The test may also give a positive result in various non-venereal treponematoses such as yaws and pinta [21]. However, some researchers argue that false-positive results are preferable to false negatives [22]. Although a false negative result, a syphilitic patient may not be treated and transmit the infection to others; A false positive serology would at least be repeated with alternative methods before a definitive diagnosis is made. In this context, we propose to adopt an inverse algorithm whereby a non-treponemal test such as VDRL or RPR could be used to document the active disease in patients with a reactive test at the point of care and then confirm it by the test fast. However, these tests are still under evaluation and not available for routine use.

Our study is very important from a public health perspective and based on our findings, we recommend the use of rapid care point procedures such as SD BIOLINE Syphilis 3.0. Screening tests as primary for the serodiagnosis of syphilis, where laboratory facilities are not available and tests to confirm the non-treponemal reactivity where TPHA cannot be done. The study was carried out in the UPFR in Immunology of the CHU-JRA. The laboratory receives many samples of diverse people from inpatients and outpatients, from various departments of hospitals.
This study has some limitations. First, the number of sample in the panel used to evaluate the kit is low. In addition, our laboratory study and evaluation was not given in the field or clinical conditions for which the test is primarily intended. In addition, the test kit was evaluated using exclusively serum samples and its performance characteristics with whole blood and plasma specimens are not determined. In addition, we don't have clinical information on the cases from which the reactive sample was obtained and therefore the performance of the test according to the stage of syphilis could not be assessed.

In conclusion, we strongly recommend the use of SD BIOLINE Syphilis 3.0 for the diagnosis of rapid on-site syphilis. Further field studies are needed before the test can be systematically implemented as a screening test.

\section{References}

The global elimination of congenital syphilis: rationale and strategy for action. Geneva: Department of Reproductive Health and Research, World Health Organization; 2007. Available from: http://www.who.int/reproductivehealth/ publications/rtis/9789241595858/en/ind ex.html [Accessed 6 February 2013].

Seña AC, White BL, Sparling PF. Novel Treponema pallidum serologic tests: A paradigm shift in syphilis screening for the 21st century. Clin Infect Dis. 2010; 51:700-8.

Allen RH, Mason MA. Cardiolipin antigens in the serodiagnosis of syphilis. Can $J$ Public Health. 1952; 43:128-35.

Gerber A, Krell S, Morenz J. Recombinant Treponema pallidum antigens in syphilis serology. Immunobiology. 1996-1997; 196: 535-49.

Rusnak JM, Butzin C, McGlasson D, Blatt 
SP. False-positive rapid plasma reagin tests in human immunodeficiency virus infection and relationship to anticardiolipin antibody and serum immunoglobulin levels. J Infect Dis. 1994; 169:1356-9.

Nandwani R, Evans DT. Are you sure it's syphilis. A review of false positive serology? Int J STD AIDS. 1995; 6:2418.

Bernard C, de Moerloose P, Tremblet C, Reber G, Didierjean L. Biological true and false serological tests for syphilis: Their relationship with anticardiolipin antibodies. Dermatologica. 1990; 180:151-3.

Mabey D, Peeling RW, Ustianowski A, Perkins MD. Diagnostics for the developing world. Nat Rev Microbiol. 2004; 2:231-40.

Young H. Syphilis: New diagnostic directions. Int J STD AIDS. 1992; 3:391-413.

Centers for Disease Control and Prevention (CDC). Discordant results from reverse sequence syphilis screening - five laboratories, United States, 2006-2010. MMWR Morb Mortal Wkly Rep. 2011; 60:133-7.

Berkowitz K, Baxi L, Fox HE. False-negative syphilis screening: The prozone phenomenon, nonimmune hydrops, and diagnosis of syphilis during pregnancy. Am J Obstet Gynecol. 1990; 163:975-7?

Ebel A, Bachelart L, Alonso JM. Evaluation of a new competitive immunoassay (BioElisa Syphilis) for screening for Treponema pallidum antibodies at various stages of syphilis. $J$ Clin Microbiol. 1998; 36:358-61.

Peeling RW, Mabey D, Herring A, Hook EW., 3rd why do we need qualityassured diagnostic tests for sexually transmitted infections? Nat Rev Microbiol. 2006; 4:909-21.

Peeling RW, Mabey D. Point-of-care tests for diagnosing infections in the developing world. Clin Microbiol Infect. 2010; 16:1062-9.

Mabey DC, Sollis KA, Kelly HA, Benzaken AS, Bitarakwate E, Changalucha J, et al., Point-of-care tests to strengthen health systems and save newborn lives: The case of syphilis. PLoS Med. 2012; 9:e1001233.

Wang LN, Yang L, Zheng HY. Clinical evaluation of four recombinant Treponema pallidum antigen-based rapid tests in the diagnosis of syphilis. Chin Med Sci J. 2007; 22:250-3.

Aboud S, Lyamuya EF, Sufi J, Haule E. Comparison of the performance of SD Bioline Syphilis 3.0 assay with the RPR test for the syphilis screening in Dar es Salaam, Tanzania. Tanzania Med J. 2006; 21:6-7.

Li J, Zheng HY, Wang LN, Liu YX, Wang XF, Liu XR. Clinical evaluation of four recombinant Treponema pallidum antigen-based rapid diagnostic tests for syphilis. $J$ Eur Acad Dermatol Venereol. 2009; 23:648-50.

Bocoum FY, Ouédraogo H, Tarnagda G, Kiba $\mathrm{A}$, Tiendrebeogo S, Bationo $\mathrm{F}$, Liestman B, Diagbouga S, Zarowsky C, Traoré RO, Kouanda S. Evaluation of the diagnostic performance and operational characteristics of four rapid immunochromatographic syphilis tests in Burkina Faso. Afr Health Sci. 2015. 15(2): 360-7. doi: 10.4314/ahs.v15i2.8.

Sato NS, de Melo CS, Zerbini LC, Silveira EP, Fagundes LJ, Ueda M. Assessment of the rapid test based on an immunochromatography technique for detecting anti-Treponema pallidum antibodies. Rev Inst Med Trop Sao Paulo. 2003; 45: 319-22.

West B, Walraven G, Morison L, Brouwers J, Bailey R. Performance of the rapid plasma reagin and the rapid syphilis screening tests in the diagnosis of 
syphilis in field conditions in rural Africa. Sex Transm Infect. 2002; 78:282-5.

Centers for Disease Control and Prevention (CDC). Syphilis testing algorithms using treponemal tests for initial screening - Four laboratories, New York City, 2005-2006. MMWR Morb Mortal Wkly Rep. 2008; 57:872-5.

Ratnam S. The laboratory diagnosis of syphilis. Can $J$ Infect Dis Med Microbial. 2005; 16:45-51.

Lien TX, Tien NT, Chanpong GF, Cuc CT, Yen VT, Soderquist R, et al., Evaluation of rapid diagnostic tests for the detection of human immunodeficiency virus types 1 and 2, hepatitis B surface antigen, and syphilis in Ho Chi Minh City, Vietnam. Am J Trop Med Hyg., 2000; 62:301-9?

\section{How to cite this article:}

Arinomenjanahary Rakotoarisoa, Herinjaka Andriamandimbisoa, Toky Randriamahazo, Jaonoharimandimby Andrianavalona, Davidra Rajaonatahiana and Andry Rasamindrakotroka. 2017. Performance of SD Bioline Syphilis 3.0 for the Diagnosis of Syphilis a UPFR in Immunology of CHU-JRA. Int.J.Curr.Microbiol.App.Sci. 6(8): 783-788. doi: https://doi.org/10.20546/ijcmas.2017.608.099 